\title{
Linear identification of a servo-pneumatic system
}

\author{
Széll Károly \\ Department of Mechatronics, Optics and Engineering \\ Informatics \\ Budapest University of Technology and Economics \\ Budapest, Hungary \\ szell@mogi.bme.hu
}

\author{
Czmerk András \\ Department of Mechatronics, Optics and Engineering \\ Informatics \\ Budapest University of Technology and Economics \\ Budapest, Hungary
}

\begin{abstract}
The identification of a nonlinear system is quite challenging for engineers. This paper presents the automatic identification of a servo-pneumatic cylinder based on a framework implemented in MATLAB. The introduced application shortens the process length of identification and gives areference model, important for controlling.
\end{abstract}

Keywords—servo-pneumaticcylinder; identification; state space

\section{INTRODUCTION}

It is a common task in engineering practice, to identify the parameters of an existing device without or with only little information about the system. The more precise model we need, the more time this process requires. Nevertheless nowadays there is always a pressure ondevelopers to be the first on the market, to have results with shorter deadlines. The lack of time claims such fast solutions like the system identification where the complex system models are not needed.

The derivation of an abstract model using conventional analysis has the following steps:

1. Choosing the parameters which best describe the system.

2. Building an abstract model representing the real system.

3. Analysis of the results and refinement of the model.

4. Generalization of the results and determining correlations.

The most problematic requirement of the steps above is to define the needed accuracy. The more accurate model we need, the more parameters and variables are needed for the building of the model and the less physical phenomenon that we can neglect. A perfect model can be beneficial, but it also has to be taken into account whether an appropriate hardware-resource is available. The identification and the calculations afterwards may take longer time than reasonable.

There are three different groups of the system-models:
1. Homolog model: scaled-down version of the real system using affinity laws.

2. Analog model: the applied physical phenomenon differs from the modelled phenomenon but the outputs of both systems are the same for the same inputs (e.g. substitution of a pneumatic system with an electrical circuit)

3. Mathematical model: description of the physical behavior of the real system by equations.

Due to the developments in computing the application of mathematical models became also widespread. It is a fast and relatively cheap solution to perform analytical tasks. The main point of this method is to define a mathematical model which describes the behavior of the real system and which is fast and accurate enough from controlling point of view. One possibility is to describe the physical phenomena directly by equations. In this case deeper knowledge is needed to find the most appropriate formula and parameters.

Another possibility to define the mathematical model is the system identification, when the model is built up based on an input signal sequence and the belonging output signal sequence. The result is a linearized empirical model. This process does not require so deep knowledge of the physical background of the system. The most important point to be defined is the number of physical elements that can store energy and thus the characteristic of the expected functions. This method gives fast results but it has to be handled a bit sceptic. This model loses the contact to the real system and can only be applied in the linearized domain. Outside of the linearized domain the response of the real system and the response of the abstract model defined by system identificationmight be different. The main disadvantage of the method is that we have no information in the abstract model about the physical background. It is not possible to change a component of the real system, and implement this modification in our mathematical model by replacing some parameters, but the whole system identification process has to be repeated for any change in the real system. This kind of abstraction cannot highlight the physical relationship of the elements of our experimental setup. 


\section{EXPERIMENTAL SETUP}

The experimental setup is a servo-pneumatic system (see Fig. 1.). The hardware elements used during the identification are listed in Table I. The aim of the experiment is to set the position of the carriage of the rodless pneumatic cylinder with the help of two $3 / 3$ proportional valves (which in this case are used as one $5 / 3$ proportional valve since they are excited simultaneously and inversely). The position is sensed by an optical incremental encoderwith $5[\mu \mathrm{m}]$ accuracy. Processing of the measured data, calculation and realization of the control signals are done by a 16-bit DSP microcontroller. Due to the different voltage levels an interface circuit is also needed. This interface circuit handles the sinusoidal signals of the incremental encoder and supplies the quadrature module of the DSP with digital signals that can be processed with the needed frequency. The interface circuit is also responsible for the voltage level shift between the output of the microcontroller (3.3 [V]) and the control signals of the proportional valves (10 [V]). The processed data is sent by UART communication from the microcontroller to the PC where aMATLAB based graphical user interface (Fig. 2.) $\operatorname{logs}$ the data for postprocessing.

This experimental setup allows the automatic identification of the rodless pneumatic cylinder which is discussed in chapter IV, but before that it is practical to analyze the elements of the system. Exact parameters and functions are not needed, only the main characteristics of the system to have an idea about the order of the system model. The necessary linearized model is described inthe next chapter.

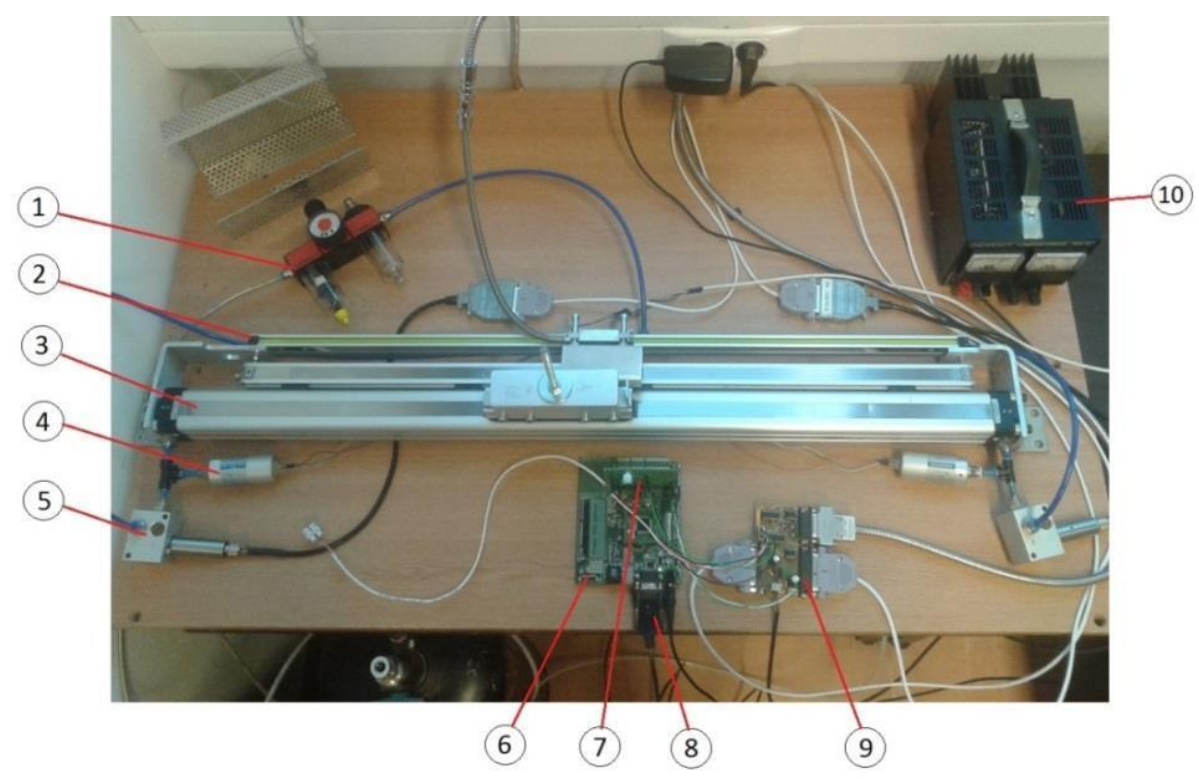

Fig. 1.Experimental setup

TABLE I. ELEMENTS OF THE EXPERIMENTAL SETUP

\begin{tabular}{|cll|}
\hline Nr. & \multicolumn{1}{c|}{ Element } & \multicolumn{1}{c|}{ Type } \\
\hline 1 & Pressure regulator & HOERBIGER SFRL-1/4 \\
2 & Incremental linear encoder & Mitutoyo AT112 \\
3 & Pneumatic cylinder & HOERBIGER P210-20 Ø32 700 \\
4 & Pressure sensor & FESTO SDE-10-10V/20mA \\
5 & $3 / 3$ proportional valve & HOERBIGER 94701 \\
6 & Microcontroller development board & DM240001 (Explorer16) \\
7 & PICtail Plus & AC164126 \\
8 & USB to serial converter & PL-2303 USB to RS232 \\
9 & Interface circuit & \\
10 & Power supply & VOLTCRAFT TNG35 \\
\hline
\end{tabular}

\section{LINEARIZED STRUCTURE}

A servo-pneumatic system is nonlinear and time-variant [15] while our identification process will result in a linear mathematical model, which has only little relationship to the physical behavior of the original real system. Our goal in this chapter is to find the main physical phenomena which define the minimal order of the system model for the needed accuracy. 
The positioning of the system depends on the pressures in the left and right chambers. The carriage is pushed by the resultant force which is the difference of the pressures acting on the left and right surfaces of the piston. The dynamic of the system is depending furthermore on the mass of the carriage and the friction between the piston and the wall of the cylinder [6-8]. The latter is a complex nonlinear phenomenon [9-10]. The Stribeck friction model is applied in the analyzed model but it is not discussed in details as the aim of the paper is a linear model.Based on these considerations the movement of the carriage can be described by a second order differential equation (1).

$$
\begin{array}{lll}
m & - & \text { mass } \\
A & - & \text { surface of the piston } \\
x & - & \text { piston position } \\
F_{f r} & - & \text { friction force } \\
p_{a} & - & \text { pressure of the left chamber } \\
p_{b} & - & \text { pressure of the right chamber } \\
F_{C} & - & \text { Coulomb friction coefficient } \\
F_{V} & - & \text { viscous friction coefficient } \\
F_{S t r} & - & \text { Stribeckfriction force } \\
K & - & \text { heat coefficient ratio } \\
\dot{m}_{i} & - & \text { mass flow } \\
R & - & \text { specific gas constant of air } \\
T & - & \text { chamber temperature } \\
g_{i} & - & \text { coefficient of the linearized variable } \\
x_{v} & - & \text { position of the valve body } \\
S_{v} & - & \text { damping coefficient of the valve } \\
\omega_{v} & - & \text { natural frequency } \\
A_{v} & - & \text { opening cross-section } \\
u & - & \text { input voltage of the valve } \\
& & \\
& &
\end{array}
$$

, where

$$
F_{f r}=F_{C} \cdot \operatorname{sign}(\dot{x})+F_{V} \cdot \dot{x}+F_{S t r}(\dot{x}, \ddot{x})
$$

The differential equation can be interpreted as a balancing of the pressures in the chambers. For example if a movement to the right direction is needed then the pressure in the left chamber has to overcome the pressure of the right chamber and also the friction force. The surplus force accelerates the carriage making the positioning possible. The linearized relationship based on these considerations can be seen in equation (3).

$$
m \cdot \ddot{x}=A \cdot\left(p_{a}-p_{b}\right)-F_{V} \cdot \dot{x}
$$

As these phenomena define the most important behavior of our experimental setup, the roots of this equation are expected to be the dominant roots of our model. Depending on the damping, these roots are real roots or complex conjugate pairswhich form is nontrivial according to the nonlinear system model.

The chamber pressure itself is also a function of several variables. The pressure might be changed in three ways. By changing the amount of air in the chamber with the help of the proportional valve. By changing the volume of the chamber when the piston is moved. By changing the temperature of the chamber. During this experiment the latter one is neglected, due to the difference of time constants for mechanical and thermal processes. Thus the chamber pressures can be described by the differential equations (4) and (5).

$$
\begin{aligned}
& \dot{p}_{a}=\frac{\kappa \cdot\left(R \cdot T_{a} \cdot \dot{m}_{a}-A \cdot \dot{x} \cdot p_{a}\right)}{A \cdot x} \\
& \dot{p}_{b}=\frac{\kappa \cdot\left(R \cdot T_{b} \cdot \dot{m}_{b}+A \cdot \dot{x} \cdot p_{b}\right)}{A \cdot x}
\end{aligned}
$$

The linearized form of the differential equations as a function of the amount of substance, the pressure and the position of the piston can be seen in equations (6) and (7).

$$
\begin{aligned}
& \dot{p}_{a}=g_{m a} \cdot \tilde{x}_{v}+g_{p a} \cdot \tilde{p}_{a}+g_{x a} \cdot \tilde{x} \\
& \dot{p}_{b}=g_{m b} \cdot \tilde{x}_{v}+g_{p b} \cdot \tilde{p}_{a}+g_{x b} \cdot \tilde{x}
\end{aligned}
$$

The mass flow of the air depends on the opening crosssection and the flow factor of the proportional valve, which is also a complex nonlinear function. Since this correlation is not a differential equation, from the point of view of our deduction, it is irrelevant.

The mass flows are controlled by two independent proportional valves. In the case of our experimental setup the valves are handled as one $5 / 3$ proportional valve as they are actuated the same time but inversely. Itsdynamic behavior can be described by a mass-spring model. This element is not likely to give the dominant conjugate pairs and thus we do not go into further details. The linearized form can be seen in equation (8).

$$
\ddot{x}_{v}+2 \cdot \varsigma_{v} \cdot \omega_{v} \cdot \dot{x}_{v}+\omega_{v}^{2} \cdot x_{v}=A_{v} \cdot \omega_{v}^{2} \cdot u
$$

As summary of the considerations above, the servopneumatic cylinder can be described by a second order differential equation of the carriage movement (3), two first order differential equations of the chamber pressures (6),(7) and a second order differential equation of the proportional valves (8). It can be concluded that for the identification at least a sixth order state space model is necessary. 

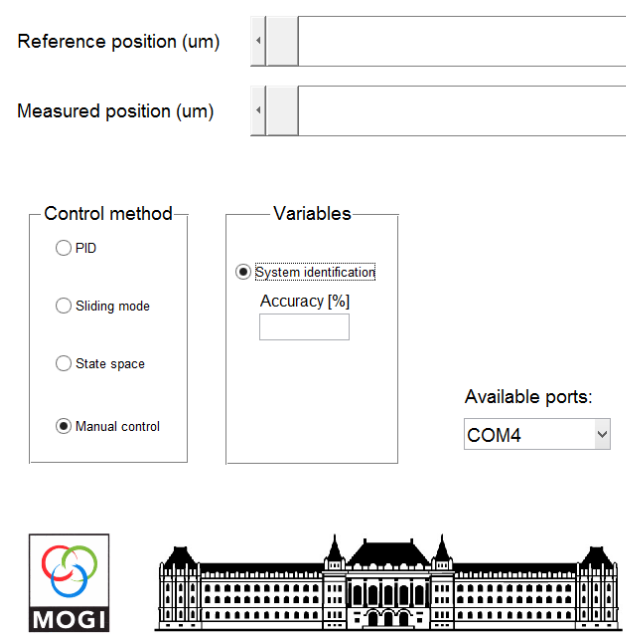

Fig. 2. MATLAB user interface

\section{PREPROCESSING OF THE MEASUREMENT DATA}

During identification the shape and type of the excitation signal, applied on the system's input has determinativeinfluences. The ideal excitation signal is the socalled persistent signal, which can be any of the conventional test signals, for example:

- impulse

- $\quad$ step signal

- white noise

- $\quad$ sum of sin signals

The measurement accuracy can be improved, if the number of samples per time unit is increased; also bydecreasing the time delay between the samples.Different signal types can achieve the same measurement accuracy,achieved with different sampling time. In our case the data was sampled with $100[\mathrm{~Hz}]$, also a sample was taken in every $10^{\text {th }}[\mathrm{ms}]$, so based on the Shannon-theorem, the useful frequency range is 50 [Hz]. Signal"data1" was used for the identification, while data2 for the verification(Fig. 3.).

The square signal was chosen, because its Fourier transform is rich in harmonics, and provides the excitation of the most system natural frequencies. The result, based on the output signal, gives a preliminary foreknowledge if there are further dominant pole/pole pairs near to the system's dominant poles. As next step if we neglect the poles that are at least three times faster than the system's dominant pole/ pole pairs, we do not make a big mistake because the effects of these poles die down faster than the system's settling time.

For the periodic signals over the window length, the discrete Fourier transform generates the complex Fourier coefficients. Thesquares ofabsolute values of these coefficients give the signal's power density. The square of the discrete Fourier transform is called the periodogram. A periodogram also can be used to estimatethe spectrum of a stochastic signal.
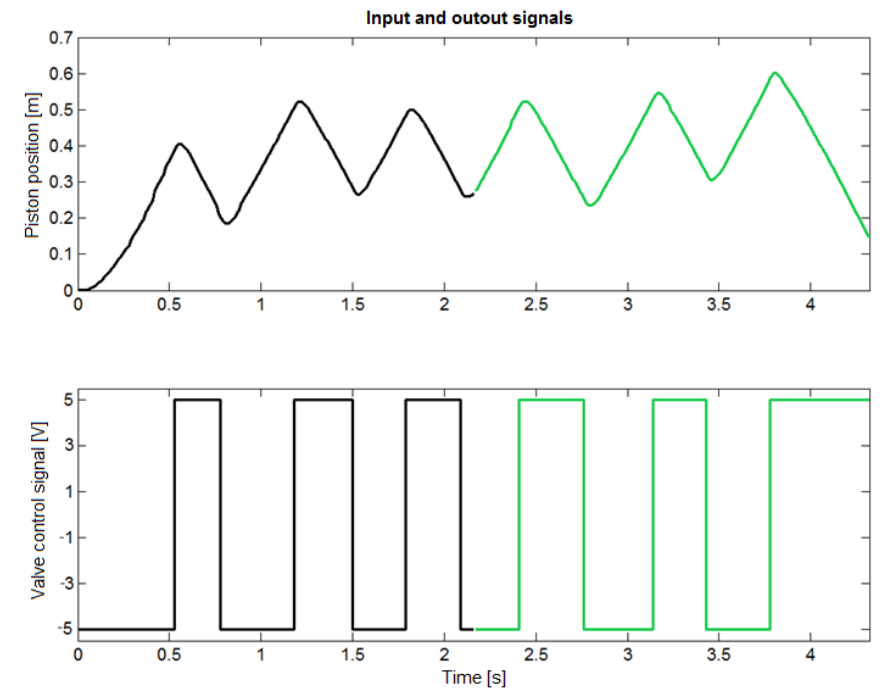

Fig. 3. Valve control signal and piston position

Before identification, during the preparation of the measured data, the following steps arenecessary:

- $\quad$ filtering higher frequency noise

- removing measurement errors

- removing DC component from the signal

As it can be seen on the measurement result, the above mentioned preparation steps can be omitted, because the measured signals are clean from noise, furthermore the starting position was $0[\mathrm{~m}]$ thanks to the initialization steps, implemented on the microcontroller.

Fordata recording and identification, a dedicated MATLAB graphical user interface was created and used (Fig. 2.). The multi-function interface is implementedwith the purpose of easing the further work with the different control methods. With the "Reference position" slider on the "Manual control" tab, we can set the PWM duty ratio of the proportional valve's control signal.Choosing "System identification" radio button on the mentioned interface tab, a $6^{\text {th }}$ order state space model identification runs automatically after collecting identification data.The accuracy of the identified model is highlighted on the same panel right after the process is done. With the "LTI view" button, the model can be analyzed in the time and frequency domain.

\section{IDENTIFICATION}

During identification a cost function is defined, which is the difference between the real system and the output of the determined model, and it is used to optimize the model parameters to reach the minimum of this function.With other words, we want from the model's output to approximate the real system's behavior accurately. This can be reached by the optimization of the model's polynomials, which is possible with the least square method, numeric optimum searching algorithms or with these combinations. 

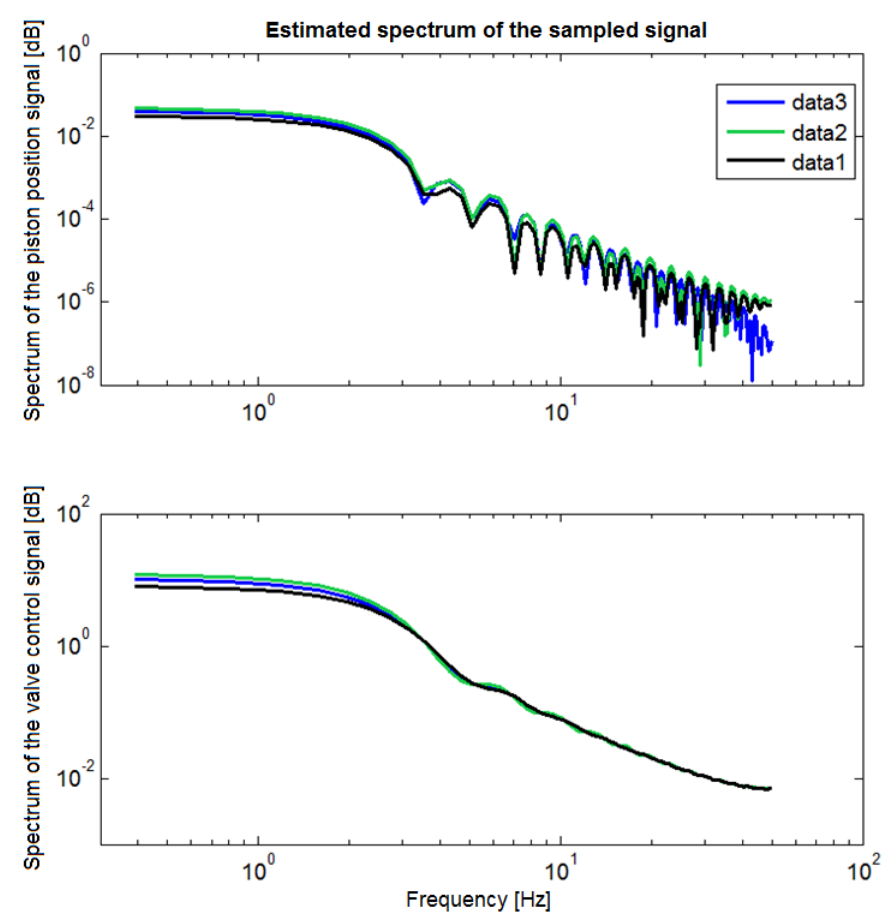

Fig. 4. Estimated spectrums of the excitation signal and piston position

The structure ofthe modeldoes not have to be the same asthestructureof the testsystem, but has to show the same behavior; otherwise, it cannot replace the system during analysis.

Identification results obtained from discrete-time linear models are expected to belong to linear, sampled continuoustime systems.

If $s_{i}$ is the pole of the continuous-time system, then it has to be mapped in the $z_{i}=e^{s_{i} T}$ pole in the sampled system's discrete-time transfer function. This means, if $z_{i}$ on the negative real axes is pole of the identified model and the multiplicity is even, than it cannot belong to a continuous-time linear system, because its $S_{i}=\frac{\ln z_{i}}{T}$ complex poles can appear only together with its $\bar{S}_{i}$ complex conjugated pairs.

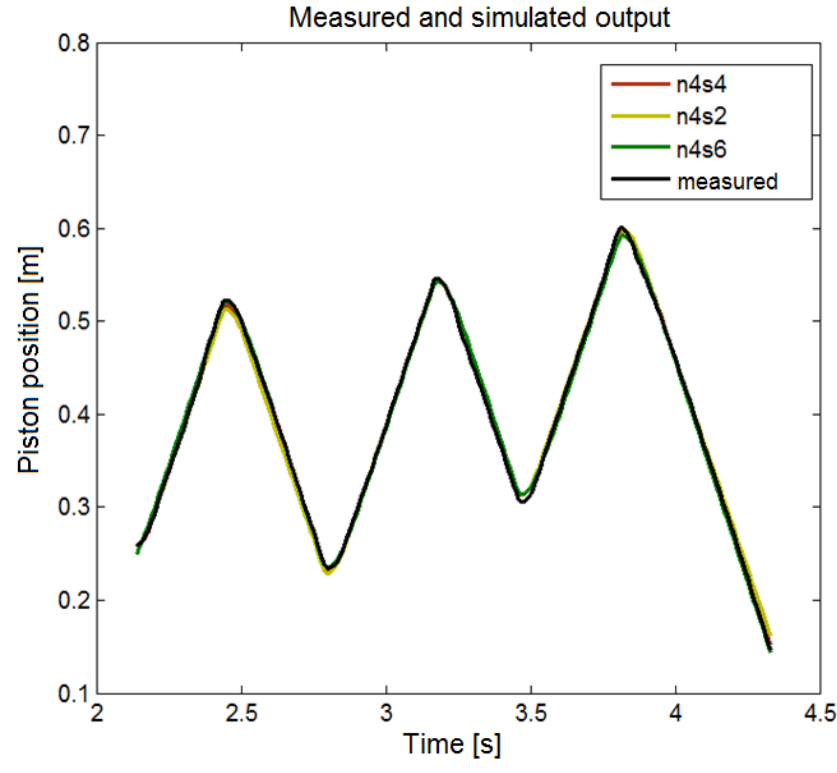

Fig. 5.Simulation results of the identified models

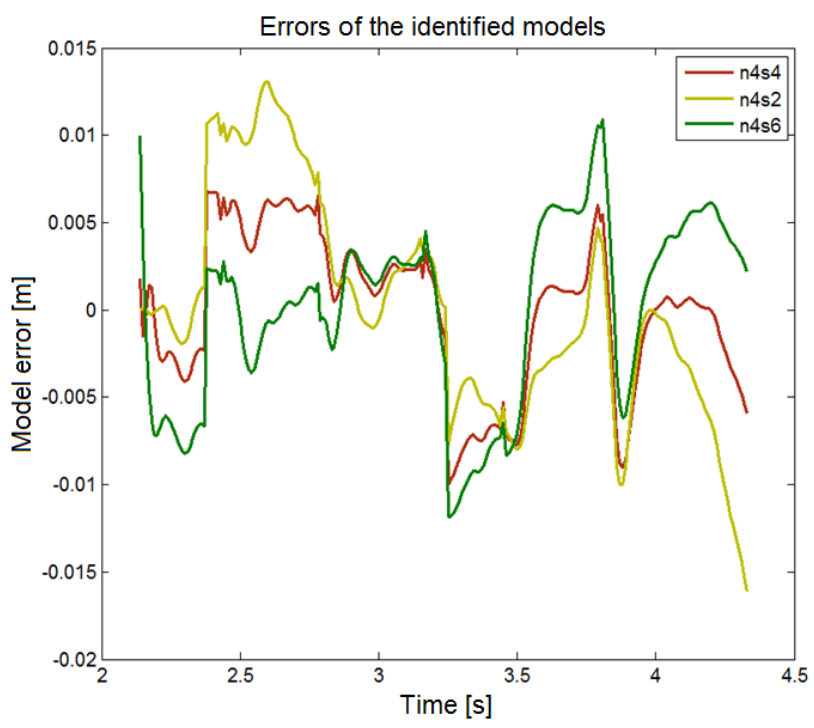

Fig. 6.Errors of the identified models

Model accuracy based on simulation:

- $\quad 4$ th order state space (n4s4): $95.83 \%$

- 2 th order state space (n4s2): $95.06 \%$

- $\quad 6$ th order state space (n4s6): $93.98 \%$ 


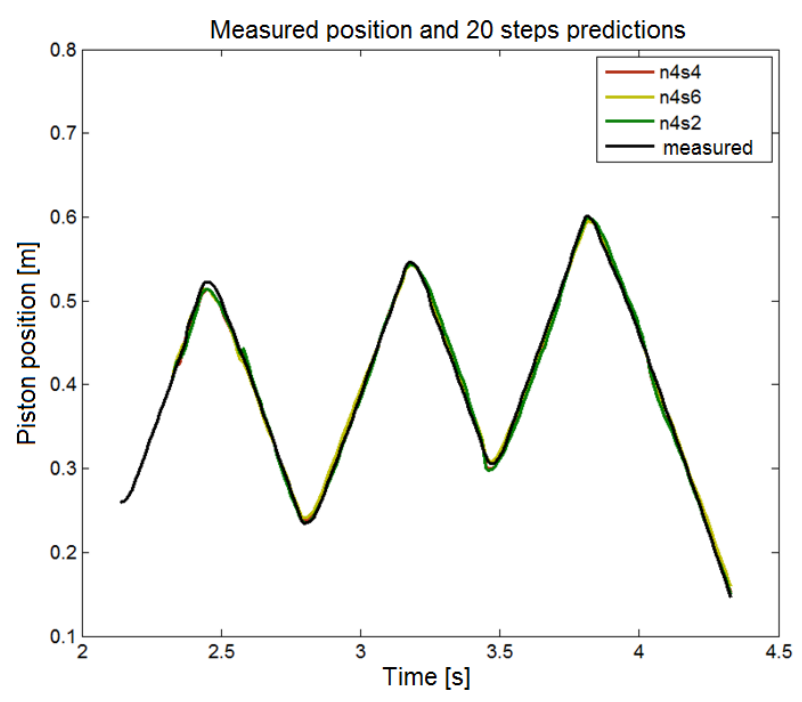

Fig. 7. 20 steps prediction of the identified models

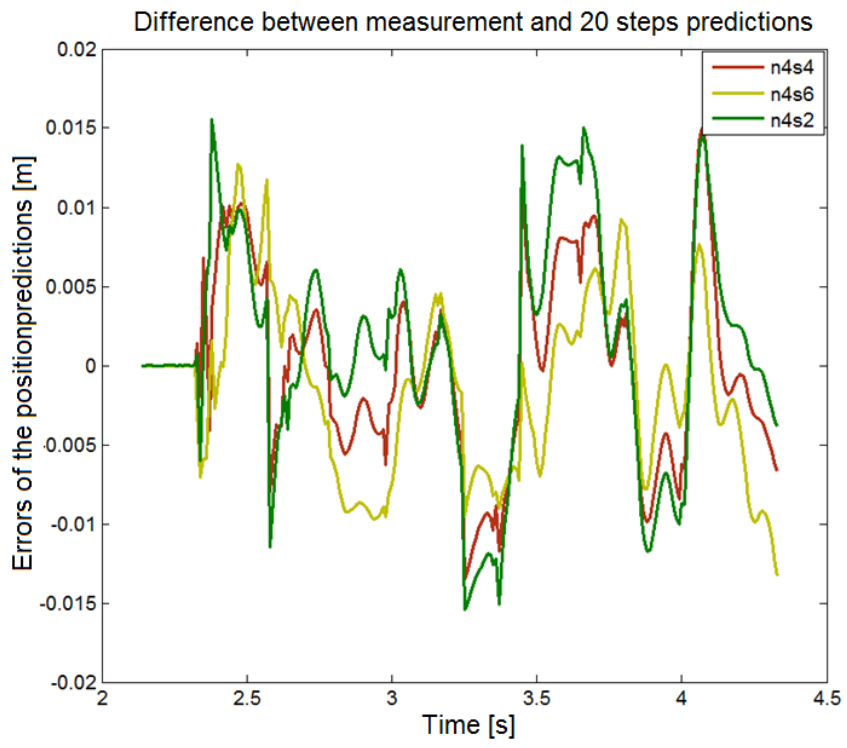

Fig. 8. Error of the 20 steps prediction of the identified models

Modell accuracy based on 20 steps prediction:

- $\quad 4$ th order state space (n4s4): $94.41 \%$

- 6th order state space (n4s6): $94.57 \%$

- 2 th order state space (n4s2): $93.19 \%$

From the identified system models, we got the best results with the linear time invariant state space models, which structure can be written in the form(9)-(10).

$$
\begin{gathered}
\dot{\boldsymbol{x}}_{(t)}=\boldsymbol{A} \boldsymbol{x}_{(t)}+\boldsymbol{B} \boldsymbol{u}_{(t)}+\boldsymbol{K} \boldsymbol{e}_{(t)} \\
\boldsymbol{y}_{(t)}=\boldsymbol{C} \boldsymbol{x}_{(t)}+\boldsymbol{D} \boldsymbol{u}_{(t)}+\boldsymbol{e}_{(t)},
\end{gathered}
$$

where A.B.C.Dare the state matrices, Kis the noise matrix, $\boldsymbol{u}_{(t)}$ is the input, $\boldsymbol{y}_{(t)}$ is the output, $\boldsymbol{x}_{(t)}$ is then ${ }^{\text {th }}$ order state vector and $\boldsymbol{e}_{(t)}$ is the noise vector.

The free tunable parameters of the system are the noise and the state matrices except the D matrix, because it is assumed, that there is no feed forward in the system, so can be neglected.

Based on the results the system can be best described by a $4^{\text {th }}$ order state space model. The determined model is acceptable, if the remaining error does not contain any structure, also it is free of any pattern, and there is not any correlation with the input or output:

- the average of white noise is null

- the process has Gaussian-distribution

- the elements of the error series and the previous input values do not correlate

The autocorrelation and the cross correlation test (Fig. 9.) can be used forthe examination of the residual error, in which the autocorrelation of the residual error and the cross correlation of the input signal and the error signal is estimated. The more similarity the remaining error has to the white noise, the more the similarity between the autocorrelation and the impulse function is. The cross correlation function is used to analyze the relationship between the input signal and the residual error. With a sufficiently accurate model there is not any correlation between the input and the error function, also the value of the cross-correlation function is near to null by any delay.
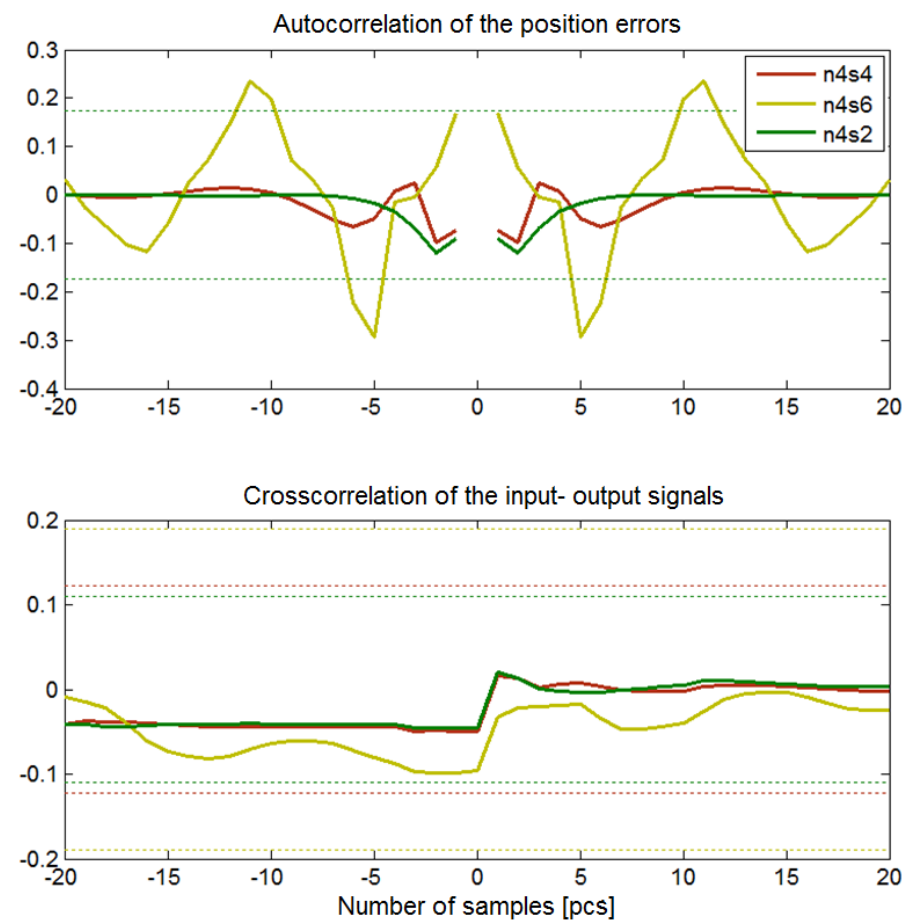

Fig. 9.Auto and cross correlation between the signals 
The zeros and the poles belonging to the identified models can be seen in Fig. 10. Note that there are not any negative real poles with the multiplicity of one, so the models can belong to a real system. Note that all the models contain an integrator, as it was expected after the real system's structure $\left(\mathrm{z}_{\mathrm{i}}=1\right)$.

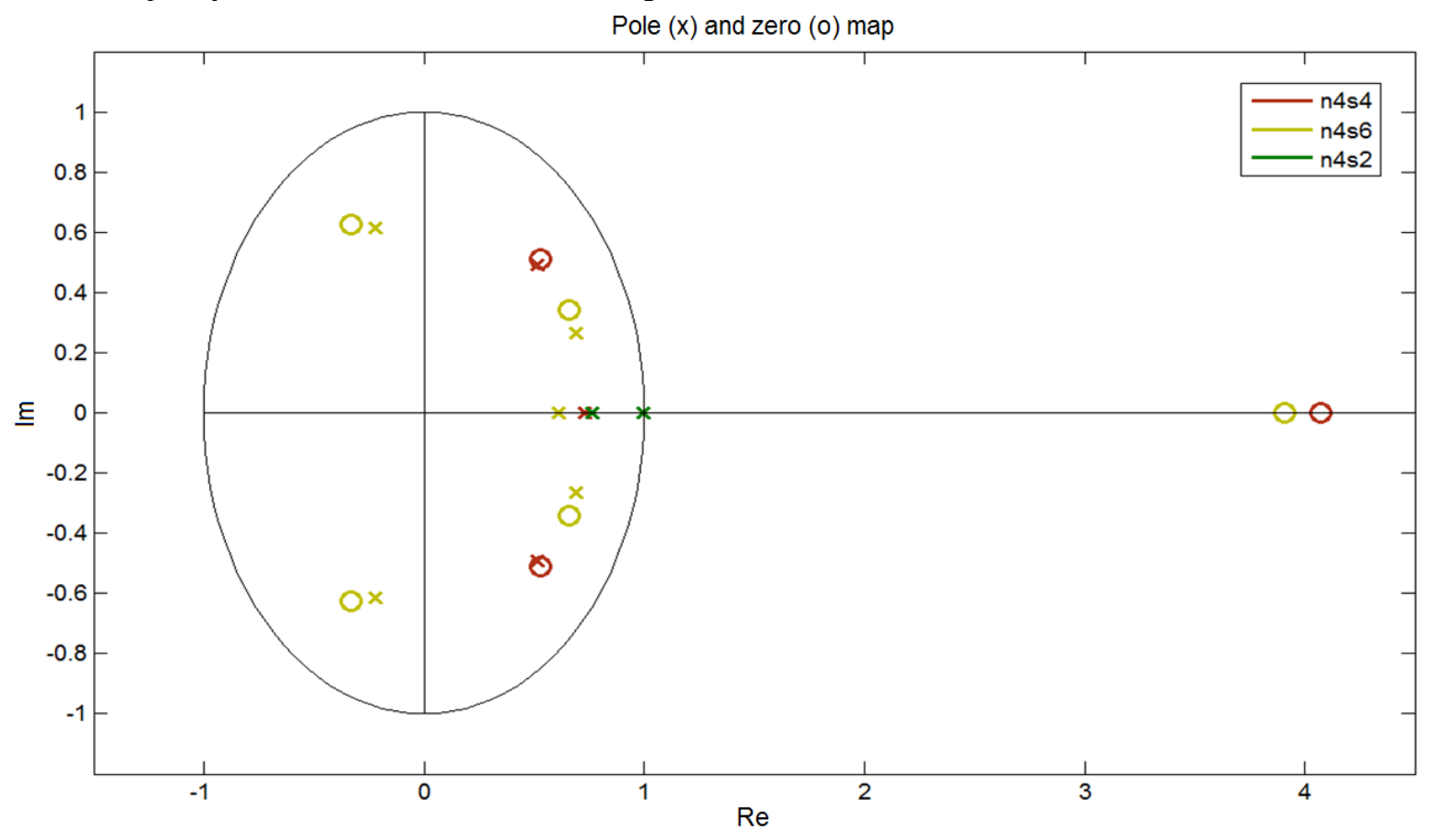

Fig. 10.Zero, pole map of the identifiacted models
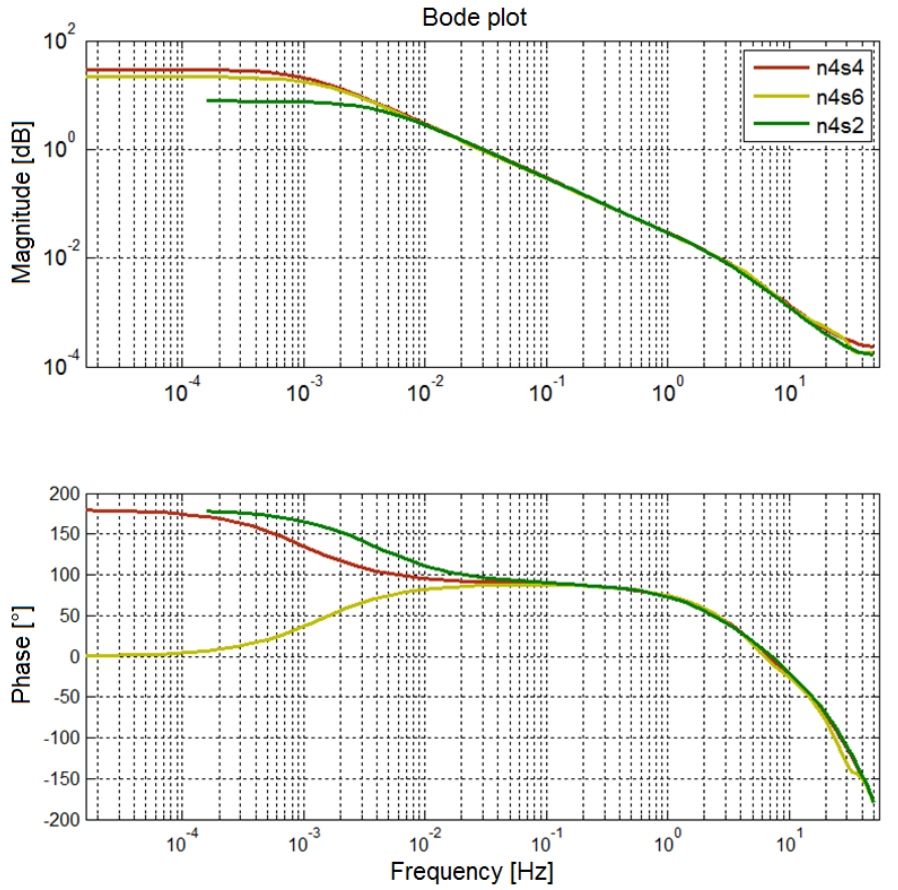

Fig. 11.Bode diagrams of the identificated models

\section{CONCLUSION}

The paper discussed the definition of the transfer function of a servo-pneumatic cylinder using two different methods. First the analytical way and then the automatic system identification based on measurement data. Based on the results the fourth order state space model has proven to be the most appropriate to describe the physical behavior of the real system.

Due to the system identification method a fast result could be obtained that is accurate enough to apply in simulations, utilize in controller development or as a reference model. Applying the model obtained by system identification, a robust state space controller with load estimation had been implemented that can properly position the real system.

\section{ACKNOWLEDGMENT}

The authors wish to thank the support to the Hungarian Automotive Technicians Education Foundation, to the Hungarian Research Fund (OTKA K100951), and the Control Research Group of HAS. The results discussed above are supported by the grant TÁMOP-4.2.2.B-10/1-- 2010-0009.

\section{REFERENCES}

[1] Ming-Chang Shih, Shy-I Tseng, "Identification and position control of a servo pneumatic cylinder", Control Engineering Practice, Volume 3 , Issue 9, September 1995, pp. 1285-1290, ISSN 0967-0661

[2] Yu-Wang Chen, Jian-Bo Yang, Chang-Chun Pan, Dong-Ling Xu, ZhiJie Zhou, "Identification of uncertain nonlinear systems: Constructing belief rule-based models", Knowledge-Based Systems, Volume 73, January 2015, Pages 124-133, ISSN 0950-7051

[3] Sárosi J., „Accurate Positioning of Pneumatic Artificial Muscle at Different Temperatures Using LabVIEW Based Sliding Mode Controller", 9th IEEE International Symposium on Applied Computational Intelligence and Informatics (SACI 2014), Timisoara, 
Recent Innovations in Mechatronics (RIiM) Vol. 2. (2015). No. 1-2.

DOI: $10.17667 /$ riim.2015.1-2/7.

Romania, 15-17 May, 2014, ISBN 978-1-4799-4693-8, ISBN 978-14799-4694-5, Pendrive, pp. 85-89

[4] M. F. Rahmat, Sy Najib Sy Salim, N. H. Sunar, Ahmad 'Athif Mohd Faudzi, Zool Hilmi Ismail and K. Huda, "Identification and non-linear control strategy for industrial pneumatic actuator", International Journal of the Physical Sciences Vol. 7(17), pp. 2565 - 2579, 23 April, 2012.

[5] Sárosi J., „New Approximation Algorithm for the Force of Fluidic Muscles", 7th IEEE International Symposium on Applied Computational Intelligence and Informatics (SACI 2012), Timisoara, Romania, 22-24 May, 2012, E-ISBN 978-1-4673-1012-3, Print ISBN 978-1-4673-1013-0, ISBN 978-1-4673-1014-7, Pendrive, pp. 229-233
[6] Piepenbrink, A., „Experimentelle Identifikation und Regelung servopneumatischer Antriebe“, Dissertation Kassel (1996)

[7] Gyeviki, J., "Dynamics of a Servopneumatic Positioning System" (2003)

[8] András Czmerk, "Dynamics of a servopneumatic drive", VII. International PhD Workshop. Gliwice, Poland, pp. 343-346., 2005.

[9] Yung-Tien Liu, Tien-Tsai Kung, Kuo-Ming Chang, Sheng-Yuan Chen, "Observer-based adaptive sliding mode control for pneumatic servo system", Precision Engineering 37, pp. 522- 530, 2013.

[10] Sy Najib Sy Salim, M.F. Rahmat, A.M. Faudzi, N.H. Sunar, Z.H. Ismail, Sharatul Izah Samsudin, "Tracking performance and disturbance rejection of pneumatic actuator system", IEEE 978-1-4673-5769-2/13, 2013 . 